\title{
Weighted Stackelberg Algorithms for Road Traffic Optimization
}

\author{
Kostas Kollias \\ kostaskollias@google.com \\ Google Research \\ Mountain View, CA, USA
}

\author{
Arun Chandrashekharapuram \\ aruncg@google.com \\ Google \\ Mountain View, CA, USA
}

\author{
Lisa Fawcett \\ lfawcett@google.com \\ Google Research \\ Mountain View, CA, USA
}

\author{
Sreenivas Gollapudi \\ sgollapu@google.com \\ Google Research \\ Mountain View, CA, USA
}

\author{
Ali Kemal Sinop \\ asinop@google.com \\ Google Research \\ Mountain View, CA, USA
}

\begin{abstract}
We study the problem faced by an online navigation platform that wishes to improve the total cost experienced by drivers in the road network, i.e., minimize the total travel time of vehicles on the streets. The platform has access to a fixed fraction of the traffic and needs to route it in a manner that improves the overall conditions in the network, both for platform users and non-users. This setting has been studied in the context of designing Stackelberg strategies for selfish routing games. An additional consideration for a routing platform in this setting is that it should ensure a positive experience for its users. The reasons for this are twofold: (a) the platform has a customer-service provider relationship with its users and (b) the users will opt out of following the platform's recommendations if they are systematically poor and the overall routing optimization effort will fail. This aspect is not explicitly addressed in standard Stackelberg algorithms and, in particular, some of them (e.g., Largest Latency First) move in the opposite direction of assigning user traffic to the most expensive paths so that the (selfish) non-user traffic can utilize the better part of the network. To address this challenge we formulate a weighted version of the Stackelberg routing problem in which the delay experienced by non-users of the platform is discounted by some parameter $\beta<1$. We study natural algorithms for this problem and provide provable guarantees in the form of constant approximation ratios for various settings. In simulations with real graphs, data-induced delay functions, and realistic demands, we exhibit that such strategies improve the experience of both platform users and independent traffic in the road network and extract the trade-off between providing high quality service to the platform's users and improving the overall total cost.
\end{abstract}

\section{CCS CONCEPTS}

- Information systems $\rightarrow$ Geographic information systems; - Theory of computation $\rightarrow$ Network games.

\section{KEYWORDS}

routing, road networks, algorithms, game theory

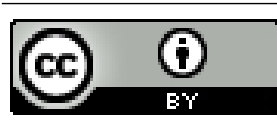

This work is licensed under a Creative Commons Attribution International 4.0 License. SIGSPATIAL '21, November 2-5, 2021, Beijing, China

(C) 2021 Copyright held by the owner/author(s).

ACM ISBN 978-1-4503-8664-7/21/11.

https://doi.org/10.1145/3474717.3483652

\section{ACM Reference Format:}

Kostas Kollias, Arun Chandrashekharapuram, Lisa Fawcett, Sreenivas Gollapudi, and Ali Kemal Sinop. 2021. Weighted Stackelberg Algorithms for Road Traffic Optimization. In 29th International Conference on Advances in Geographic Information Systems (SIGSPATIAL '21), November 2-5, 2021, Beijing, China. ACM, New York, NY, USA, 12 pages. https://doi.org/10.1145/ 3474717.3483652

\section{INTRODUCTION}

The problem of managing road traffic congestion is one that impacts billions of people living in urban areas around the world. It is then natural that understanding the inefficiencies induced by the behavior of drivers and designing methods to contain them are topics that have received significant attention in the scientific literature. One of the most popular and established models for studying urban traffic, both in theoretical $[13,16,20]$ and practical $[3,5,8,21]$ work, is the Wardrop flow model. In the Wardrop model traffic is modeled as a collection of flows in a graph $G=(V, E)$. Each edge (road segment) $e \in E$ in this graph (road network) is endowed with a latency function $l_{e}(x)$ that gives the delay $l_{e}\left(f_{e}\right)$ experienced by traffic on that particular road segment when the total flow of vehicles passing through it is $f_{e}$.

Vehicles in this framework are modeled as non-atomic particles of the flow and it is assumed that they will route themselves so that all particles use a shortest path between their respective origins and destinations, thus reaching what is termed the Wardrop equilibrium. It is known that such equilibria can be suboptimal in terms of the social cost, which is typically the total delay experienced by the traffic in the network. The headroom between the traffic equilibrium and the overall optimal routing solution has been explored theoretically in [16] and in real world instances in [5]. The former study shows that the equilibrium solution can be, in worst case networks, 1.333 times more costly than the optimal when the delay functions are affine, i.e., $l_{e}(x)=a_{e} x+b_{e}$, and 2.151 times more costly than the optimal when the delay functions are of the form $l_{e}(x)=a_{e} x^{4}+b_{e}$, which has been established as the standard cost model by the Bureau of Public Roads [10,17]. The latter study, using the $l_{e}(x)=a_{e} x^{4}+b_{e}$ cost model, found that the average inefficiency due to selfish driving behavior over five real metro road networks was approximately $7 \%$.

Methods to alleviate the inefficiencies induced by selfish behavior in the road network include marginal cost taxes $[6,12,18,19]$, which form the theoretical basis for dynamic tolls mechanisms applied today, and Stackelberg routing $[4,14,19]$. Stackelberg routing 
focuses on a centralized entity that is able to dictate routes to a fixed fraction of the traffic. This entity can then route the controlled flow collectively in a manner such that, even after the remaining flow responds with selfish route selections, the total cost is low.

The Stackelberg framework has natural similarities with online navigation platforms. Such platforms have seen increased popularity in the past decade and have reached a point of having access to a non-trivial fraction of the traffic in certain urban areas. In their current instantiations, navigation platforms will recommend to each user her fastest route according to current conditions in the road network. In this regard, the platforms contribute to the flow reaching a Wardrop equilibrium, as described above. In this work we focus on Stackelberg algorithms for an online navigation platform that wishes to instead route traffic in a network-wide optimal manner.

A disconnect between the Stackelberg framework and an online navigation platform is that such a platform does not exert control over the traffic it has access to, but rather it recommends routes to the users. Moreover, the users have a customer-service provider relationship with the platform. In this regard, the Stackelberg routing algorithm needs to ensure a positive experience for the platform's users. This is not explicitly addressed in Stackelberg routing algorithms, instead, some of the standard algorithms, such as Largest Latency First $[14,19]$, move in the direction of assigning user traffic on the most expensive paths of the optimal solution so that the selfish non-user traffic can utilize the better part of the network. This feature of the algorithm becomes a weakness in the context described above. To account for this aspect of the problem, in this work we study the weighted Stackelberg routing setting in which the travel time of non-users is discounted by a positive parameter $\beta<1$.

\subsection{Our Contributions}

1.1.1 Theoretical Analysis. We formulate the weighted Stackelberg routing problem which applies a discount parameter $\beta<1$ on the cost of non-users in the objective function. As we see in our experimental evaluation, algorithms that utilize this formulation can help address significant drawbacks of standard solutions for the problem. We show that the problem is approximable within constant factors by proving theoretical worst-case guarantees for certain algorithms. Our bounds are parameterized by (a) the fraction $\alpha$ of the traffic using the platform, (b) the non-user travel time discount parameter $\beta$, and (c) the degree $d$ of the cost functions $l_{e}(x)=a_{e} x^{d}+b_{e}$. For the case of $d=1$, we analytically show how to extract the upper bound for general $\alpha, \beta$ and explicitly compute it for specific values of interest as an example.

1.1.2 Data Analysis. We use real graphs to analyze the performance of different algorithms. In terms of delay functions, we use real data and fit to the functional form $l_{e}(x)=a_{e} x^{4}+b_{e}$, which is the established cost model as per the Bureau of Public Roads [10] and is also the functional form used in other real world studies of the Wardrop traffic model $[3,5]$. We run experimental evaluations for seven algorithms across four cities (New York City, Tokyo, Bangalore, and Delhi). Our contributions are as follows:
- We present what is, to the best of our knowledge, the first data-driven evaluation of standard and novel Stackelberg algorithms for routing in road networks.

- We quantify the trade-off between the excess travel time experienced by the platform users (i.e., the Stackelberg flow cost) and the improvement in total travel time in the network (i.e., the total cost) across seven algorithms.

- We show that standard algorithms suffer in terms of the cost experienced by the platform's user which can be significantly higher than the cost of non-users and even higher than the cost they were experiencing before the intervention of the platform. In fact this is true across all four cities for realistic platform penetration rates. In this regard we show that users have an incentive to opt out of using the platform making traditional algorithms impractical.

- We show that our novel algorithms that utilize the weighted formulation of the Stackelberg problem perform well with respect to the trade-off between the total cost and the user excess cost discussed above.

\subsection{Related Work}

The first Stackelberg routing algorithms in simple networks with parallel roads were studied in [14], where it was shown that the Stackelberg routing problem is NP-Hard (even in these simple graphs) and that two algorithms, called Scale and Largest Latency First, achieve constant factor approximations. The analysis was generalized to arbitrary networks in [19], which obtained a constant factor approximation for the case of general networks and polynomial delay functions, and also improved bounds for the case of parallel links. The bounds for polynomials were improved in [4], where the authors also proved that, with unconstrained delay functions, no constant bound is possible.

In terms of data-driven studies, $[5,21]$ study the inefficiency of equilibria and other parameters in multiple cities. The works in $[3,8]$ study the inefficiency of equilibria by refining theoretical definitions based on real world data in Singapore. Our work combines the above two lines of work (theory and data-driven) in that we design Stackelberg routing algorithms and then test both our and previous algorithms in real world graphs to evaluate the impact of Stackelberg solutions in routing.

Other studies of game-theoretic equilibria and their efficiency compared against an optimal solution in geo systems include the parking assignment problem [1], ride sharing for taxi passengers[7], and organizing geo-social events [9].

\section{PRELIMINARIES}

The Wardrop routing model is defined on a directed graph $G=$ $(V, E)$ which models the road network. Each edge $e$ has a delay function of the form

$$
l_{e}(x)=a_{e} x^{d}+b_{e}
$$

for a positive integer $d$, which gives the delay experienced by traffic on that edge when the flow through it is $x$. For every pair of nodes $s, t$, there exists a flow $r_{s, t}$ of traffic that wishes to travel from $s$ to $t$ and an $\alpha$ fraction of that flow is routed by recommendations of a central planner (e.g., a navigation platform). We refer to $\alpha$ as the 
penetration rate of the platform. This means we assume the central planner has uniform control of the traffic over all origin-destination pairs. The central planner determines how these $\alpha$ fractions of the flow are routed, assigning flow $g_{P}$ to path $P$ so that

$$
\sum_{P: s-t \text { path }} g_{P}=\alpha r_{s, t}
$$

inducing flow $g_{e}=\sum_{P: e \in P} g_{P}$ on each edge $e$. The remaining flow $h$ is a Wardrop equilibrium, meaning that, for every pair of $s-t$ paths $P, P^{\prime}$ with $h_{P}>0$, we have

$$
\sum_{e \in P} l_{e}\left(g_{e}+h_{e}\right) \leq \sum_{e \in P^{\prime}} l_{e}\left(g_{e}+h_{e}\right) .
$$

This implies that if two $s$ - $t$ paths carry positive flow then they have the same delay, whereas $s-t$ paths that don't carry any $s$ - $t$ flow can only be more expensive.

A known and useful fact about equilibrium flows, which follows by definition, is the variational inequality, which for flow $h$ can be written as:

$$
\sum_{e \in E} h_{e} \cdot l_{e}\left(g_{e}+h_{e}\right) \leq \sum_{e \in E} h_{e}^{\prime} \cdot l_{e}\left(g_{e}+h_{e}\right),
$$

for any flow $h^{\prime}$ of size $(1-\alpha) r_{s, t}$ for every pair $s, t$.

In this context our goal is to determine flow $g$, which we call the Stackelberg flow, so that, after flow $h$ is routed according to the equilibrium condition, we minimize the weighted social cost:

$$
C^{\beta}(g, h)=\sum_{e \in E} g_{e} \cdot l_{e}\left(g_{e}+h_{e}\right)+\beta h_{e} \cdot l_{e}\left(g_{e}+h_{e}\right),
$$

with $\beta \in[0,1]$ a discount parameter for the delay of the selfish traffic that is routed independently. Note that $\beta=1$ recovers the standard Stackelberg routing problem.

\section{1 (Weighted) Stackelberg Routing Example}

In this section we present the differences between standard Stackelberg routing and the weighted version we study in this work. Consider the simple and well-known Pigou network. In this network, there are only two nodes in the graph, $s$ and $t$, and there is one unit of traffic that wishes to travel from $s$ to $t$. There exist two parallel edges from $s$ to $t$. The first one, edge $e$, has a delay function $l_{e}(x)=x$ and the second one, edge $e^{\prime}$, has a delay $l_{e^{\prime}}(x)=1$.

2.1.1 Equilibrium. Observe that in the Pigou instance, independent vehicles will always prefer to travel on edge $e$, which has a cost less than 1 if any traffic is on edge $e^{\prime}$. Hence, we know that flow $h$ will route its whole $1-\alpha$ on edge $e$, independent of the decision we make for the Stackelberg flow $g$. Hence $h_{e}=1-\alpha$ and $h_{e^{\prime}}=0$. Note, that in the independent equilibrium case where $\alpha=0$, we get that all traffic suffers a unit cost, which is the worst possible for this instance.

2.1.2 Stackelberg Routing. In terms of Stackelberg solutions, in the standard (unweighted) version of the problem, we wish to minimize the total delay. The delay on edge $e$ is equal to the flow on it multiplied by the delay function:

$$
\left(g_{e}+h_{e}\right) \cdot l_{e}\left(g_{e}+h_{e}\right)=\left(g_{e}+1-\alpha\right)^{2} .
$$

The delay on edge $e^{\prime}$ is similarly:

$$
\left(g_{e^{\prime}}+h_{e^{\prime}}\right) \cdot l_{e^{\prime}}\left(g_{e^{\prime}}+h_{e^{\prime}}\right)=1-g_{e} .
$$

Then, we can optimize the expression for the total delay over all values of $g_{e} \in[0, \alpha]$ :

$$
\left(g_{e}+1-\alpha\right)^{2}+\left(\alpha-g_{e}\right)
$$

which for $\alpha=1 / 2$ is minimized at $g_{e}=0$. Observe then that all platform users suffer a delay of 1 vs a delay of 0.5 for non-users (a $100 \%$ increase), for an average of 0.75 . This example exhibits why standard Stackelberg routing can be non-applicable in online navigation platforms. Users suffer twice the cost of non-users and have a strong incentive to opt out of using the platform.

2.1.3 Weighted Stackelberg Routing. In weighted Stackelberg routing, the delay of non-users is discounted by parameter $\beta$. The user delay on edge $e$ is $g_{e} \cdot l_{e}\left(g_{e}+1-\alpha\right)$ and the user delay on edge $e^{\prime}$ is $\left(\alpha-g_{e}\right) \cdot l_{e^{\prime}}\left(\alpha-g_{e}\right)$. The discounted non-user delay on edge $e^{\prime}$ is $\beta(1-\alpha) \cdot l_{e}\left(g_{e}+1-\alpha\right)$. There are no non-users on edge $e^{\prime}$, as argued above. These give a total delay of:

$$
\begin{aligned}
g_{e} \cdot l_{e}\left(g_{e}+1-\alpha\right) & +\beta(1-\alpha) \cdot l_{e}\left(g_{e}+1-\alpha\right) \\
& +\left(\alpha-g_{e}\right) \cdot l_{e^{\prime}}\left(\alpha-g_{e}\right),
\end{aligned}
$$

which for $\alpha=\beta=1 / 2$ is optimized at $g_{e}=1 / 8$. For a more extreme value of $\beta=0$, the problem optimizes for:

$$
g_{e} \cdot l_{e}\left(g_{e}+1-\alpha\right)+\left(\alpha-g_{e}\right) \cdot l_{e^{\prime}}\left(\alpha-g_{e}\right),
$$

which for $\alpha=1 / 2$ is optimized at $g_{e}=1 / 4$, to give an average delay of 0.875 for platform users and 0.75 for non-users. The overall average delay is 0.8125 , which is an increase over the 0.75 achieved by the optimal (unweighted) Stackelberg policy, however the user delay is a $16.67 \%$ increase over the delay of non-users, as opposed to a $100 \%$ increase in the optimal (unweighted) Stackelberg policy. Hence, it is a more realistic outcome, with a relatively small degradation in performance.

In what follows we present algorithms with provable guarantees for the weighted Stackelberg routing problem. In our experimental evaluation we explore parameters for $\alpha$ and $\beta$ that yield a good trade-off between (a) how the (unweighted) total delay increases over standard Stackelberg algorithms and (b) how close the user and non-user average delays are. In other words, we seek algorithms which yield outcomes the users are willing to comply with and compare well against the (non-realistic) Stackelberg solutions in terms of total delay.

\section{ALGORITHMS AND PERFORMANCE GUARANTEES}

Computing the optimal assignment for the Stackelberg flow is an NP-Hard problem even in very simple networks of parallel links [14]. Approximation algorithms for the problem typically consist of two stages. In the first one a solution that assumes control over the whole traffic is computed. Then this initial solution is used to assign the user (Stackelberg) flow in the network. Finally, the non-user traffic responds to the Stackelberg flow selfishly, i.e., by finding fastest paths for its particles.

Our algorithms also proceed in two such stages. In the first stage, the algorithm computes a near optimal assignment of both the user flow $g$ and the non-user flow $h$, in terms of the weighted objective function $C^{\beta}(g, h)$. We write $(\hat{g}, \hat{h})$ for this initial solution. In the 
second stage, the algorithm determines how to route the user flow $g$, using the $(\hat{g}, \hat{h})$. We present several ways to make this decision.

\subsection{Initial Solution Computation}

3.1.1 (Heuristic) Solution. The following mathematical program computes the optimal solution for the weighted Stackelberg objective function. Note that the program provides the optimal solution assuming both the user and non-user flows are centrally routed, and not that the non-user flow will play a Wardrop equilibrium. The decision variables of the program give the user flow $g_{e}^{s, t}$ and the non-user flow $h_{e}^{s, t}$ that originate at $s$, travel to $t$, and pass through edge $e$.

$$
\begin{aligned}
& \text { Minimize } \quad \sum_{e \in E}\left(g_{e}+\beta h_{e}\right) \cdot l_{e}\left(g_{e}+h_{e}\right) \\
& \text { Subject to } g_{e}=\sum_{s \in V} \sum_{t \in V} g_{e}^{s, t}, \quad \forall e \in E \\
& \alpha r_{s, t}=\sum_{e \text { out of } s} g_{e}^{s, t}, \quad \forall s \in V, t \in V \\
& \alpha r_{s, t}=\sum_{e \text { into } t} g_{e}^{s, t}, \quad \forall s \in V, t \in V \\
& \sum_{e \text { out of } v} g_{e}^{s, t}=\sum_{e \text { into } v} g_{e}^{s, t}, \quad \forall v \in V, v \neq s, v \neq t \\
& h_{e}=\sum_{s \in V} \sum_{t \in V} h_{e}^{s, t}, \quad \forall e \in E \\
& (1-\alpha) r_{s, t}=\sum_{e \text { out of } s} h_{e}^{s, t}, \quad \forall s \in V, t \in V \\
& (1-\alpha) r_{s, t}=\sum_{e \text { into } t} h_{e}^{s, t}, \quad \forall s \in V, t \in V \\
& \sum_{e \text { out of } v} h_{e}^{s, t}=\sum_{e \text { into } v} h_{e}^{s, t}, \quad \forall v \in V, v \neq s, v \neq t \\
& g_{e}^{s, t} \geq 0, \quad \forall s \in V, t \in V, e \in E \\
& h_{e}^{s, t} \geq 0, \quad \forall s \in V, t \in V, e \in E
\end{aligned}
$$

The constraints ensure flow conservation and that we route the full user flow for $g$ and the full non-user flow for $h$ over all $s-t$ pairs. Given that the constraints are linear, the above optimization problem can be solved efficiently if the objective function is convex. Unfortunately the objective is not a convex function when $\beta<$ 1 and, hence, the problem can't be solved efficiently via convex programming.

One can still obtain, through local search, a heuristic solution for this program which can then be used as the initial solution for the Stackelberg routing stage. In fact, some of the algorithms we describe later in the section do exactly that. We give a description of our local search procedure in Algorithm 1 of Section 4.2. Other algorithms instead use the optimal solution $\left(g^{*}, h^{*}\right)$ for the unweighted version of the objective function, i.e., the optimal solution for $\beta=1$ (which can be computed in polynomial time as we observe next).

3.1.2 Approximation via the Unweighted Optimal Solution. Note that in this case user and non-user traffic are interchangeable. The program below solves for finding flow $f$ which we will later decompose into $g+h$ :

$$
\begin{array}{lll}
\text { Minimize } & \sum_{e \in E} f_{e} \cdot l_{e}\left(f_{e}\right) & \\
\text { Subject to } & f_{e}=\sum_{s \in V} \sum_{t \in V} f_{e}^{s, t}, & \forall e \in E \\
& r_{s, t}=\sum_{e \text { out of } s} f_{e}^{s, t}, & \forall s \in V, t \in V \\
& r_{s, t}=\sum_{e \text { into } t} f_{e}^{s, t}, & \forall s \in V, t \in V \\
& \sum_{e \text { out of } v} f_{e}^{s, t}=\sum_{e \text { into } v} f_{e}^{s, t}, & \forall v \in V, v \neq s, v \neq t \\
f_{e}^{s, t} \geq 0, & \forall s \in V, t \in V, e \in E
\end{array}
$$

Note that for our $l_{e}(x)=a_{e} x^{d}+b_{e}$, the objective function is now convex.

\subsection{Stackelberg Routing Algorithms}

To compute the Stackelberg (user) flow, we will typically decompose the initial solution into a user and a non-user flow and then apply this information to determine how we will route platform users.

3.2.1 Decomposing the Unweighted Optimal Solution. Given the values of the $f_{e}^{s, t}$ variables, we may use standard flow decomposition to decompose the $f^{s, t}$ flow into a set of paths $\mathcal{P}^{s, t}$ with each $P \in \mathcal{P}^{s, t}$ having flow $f_{P}^{s, t}$. We consider three distinct ways of splitting the flow $f_{P}^{s, t}$ into $g_{P}^{s, t}+h_{P}^{s, t}$. In each one, we need to ensure that $\sum_{P \in \mathcal{P} s, t} g_{P}^{s, t}=\alpha r_{s, t}$ and $\sum_{P \in \mathcal{P} s, t} h_{P}^{s, t}=(1-\alpha) r_{s, t}$. Below we explain how each algorithm determines the user flow $g$. Then we get the non-user flow as $h=f-g$.

Algorithm: SLF (Smallest Latency First). This algorithm starts assigning the user flow $g$ on the cheapest paths in $\mathcal{P}^{s, t}$. Formally, the algorithm sorts the paths as $P_{1}, P_{2}, \ldots, P_{|\mathcal{P} s, t|}$ from smallest to largest latency in $f$ and sets:

$$
g_{P_{i}}^{s, t}=\max \left\{0, \min \left\{f_{P_{i}}^{s, t}, \alpha r_{s, t}-\sum_{j=1}^{i-1} g_{P_{j}}^{s, t}\right\}\right\} \text {. }
$$

Note that SLF is in contrast to the typically used LLF (Largest Latency First) which begins assigning $g$ using the most expensive paths as follows.

Algorithm: LLF (Largest Latency First). The algorithm sorts the paths as $P_{1}, P_{2}, \ldots, P_{|\mathcal{P} s, t|}$ from largest to smallest latency in $f$ and uses the same formula as SLF to assign flow.

Algorithm: SCALE. This algorithm sets $g_{P}^{s, t}=\alpha f_{P}^{s, t}$.

Algorithms SCALE and LLF have been defined and used in previous work $[14,19]$.

3.2.2 Decomposing the Heuristic Weighted Solution. Similarly, we may decompose a solution obtained heuristically (e.g., via local search) for the weighted objective function into sets of paths with positive flow. Note that such a solution is already broken into user and non-user flow, a fact that we use in our last algorithm. The 
algorithms that route user flow using a heuristic initial solution $(g, h)$ for the weighted objective functions are as follows.

Algorithm: $\beta$-SLF ( $\beta$-Smallest Latency First). Set $f=g+h$ and apply SLF on $f$.

Algorithm: $\beta$-LLF ( $\beta$-Largest Latency First). Set $f=g+h$ and apply LLF on $f$.

Algorithm: $\beta$-SCALE. Set $f=g+h$ and apply Scale on $f$.

Algorithm: $\beta$-Direcr. This algorithm simply uses the user flow as computed by the heuristic optimization algorithm.

\subsection{Non-User Flow Prediction}

In the preceding sections we discussed how to compute an initial solution and how to use it to route the user flow. For the purposes of obtaining measurements in our experimental section, we need to be able to predict how the non-user flow will act. Recall that this independent traffic is assumed to be selfish and that it will be a Wardrop equilibrium over the user flow. It is known that the Wardrop equilibrium $h$ minimizes the potential function:

$$
\Phi(h)=\sum_{e \in E} \int_{0}^{h_{e}} l_{e}(y) d y .
$$

Hence to compute the non-user flow we may take the following steps. First we calibrate the delay functions to account for the presence of the user flow $g$, i.e., we define:

$$
\hat{l}_{e}(x)=l_{e}\left(x+g_{e}\right)=a_{e}\left(x+g_{e}\right)^{d}+b_{e},
$$

and then get the potential function:

$$
\Phi(f)=\sum_{e \in E} \int_{0}^{f_{e}} \hat{l}_{e}(y) d y=\sum_{e \in E} \frac{a_{e}}{d+1}\left(f_{e}+g_{e}\right)^{d+1}+b_{e} f_{e} .
$$

Note that the potential function is convex. Hence, replacing the objective of the convex program in Section 3.1.2 with the potential function allows us to solve for the non-user flow.

\subsection{Theoretical Guarantees}

The main analytical result of this section is that the weighted Stackelberg routing problem can be solved within a constant factor approximation. We provide analytical results for the algorithms that route the user flow as the result $g^{*}$ given by the SLF and SCALE decompositions of the unweighted optimal solution $f^{*}$. In our experimental evaluation we study the performance of all 7 algorithms described above in real road networks.

Lemma 3.1. For SLF and SCALE:

$$
\sum_{e \in E} h_{e}^{*} \cdot l_{e}\left(g_{e}^{*}+h_{e}^{*}\right) \geq(1-\alpha) \cdot C^{1}\left(g^{*}, h^{*}\right)
$$

Proof. For SLF, the lemma follows by the fact that the non-user flow is a $1-\alpha$ fraction for each $s-t$ pair and the user flow is assigned on the cheapest paths. Hence, the non-user flow suffers a cost that is at least proportional to its size.

For SCALE the expression holds with equality by definition: The non-user flow is a $1-\alpha$ fraction of the total flow on every path.
We present a second technical lemma before presenting the approximation ratio of $\left(g^{*}, h^{*}\right)$ against the optimal solution for the weighted objective function.

LEMMA 3.2.

$$
C^{1}(g, h) \leq \frac{C^{\beta}(g, h)}{\beta} .
$$

Proof. We get:

$$
\begin{aligned}
C^{1}(g, h) & =\sum_{e \in E}\left(g_{e}+h_{e}\right) \cdot l_{e}\left(g_{e}+h_{e}\right) \\
& \leq \frac{\sum_{e \in E}\left(\beta g_{e}+\beta h_{e}\right) \cdot l_{e}\left(g_{e}+h_{e}\right)}{\beta} \\
& \leq \frac{\sum_{e \in E}\left(g_{e}+\beta h_{e}\right) \cdot l_{e}\left(g_{e}+h_{e}\right)}{\beta}=\frac{C^{\beta}(g, h)}{\beta},
\end{aligned}
$$

which completes the proof.

We now show that the weighted objective value $C^{\beta}\left(g^{*}, h^{*}\right)$ of this solution is within a constant of the optimal.

TheOREM 3.3. Let $\left(g^{*}, h^{*}\right)$ be a SCALE or SLF decomposition of the optimal solution with respect to objective $C^{1}(g, h)$ and let $\left(g^{\beta}, h^{\beta}\right)$ be the optimal solution with respect to objective $C^{\beta}(g, h)$. Then:

$$
C^{\beta}\left(g^{*}, h^{*}\right) \leq\left(1+\alpha \frac{1-\beta}{\beta}\right) \cdot C^{\beta}\left(g^{\beta}, h^{\beta}\right) .
$$

Proof. Let:

$$
C^{g^{*}}=\sum_{e \in E} g_{e}^{*} \cdot l_{e}\left(g_{e}^{*}+h_{e}^{*}\right)
$$

and:

$$
C^{h^{*}}=\sum_{e \in E} h_{e}^{*} \cdot l_{e}\left(g_{e}^{*}+h_{e}^{*}\right)
$$

be the total travel times (costs) of users and non-users respectively. We get:

$$
\begin{aligned}
\frac{C^{\beta}\left(g^{*}, h^{*}\right)}{C^{1}\left(g^{*}, h^{*}\right)} & =\frac{C^{g^{*}}+\beta C^{h^{*}}}{C^{1}\left(g^{*}, h^{*}\right)}=\frac{C^{1}\left(g^{*}, h^{*}\right)-(1-\beta) C^{h^{*}}}{C^{1}\left(g^{*}, h^{*}\right)} \\
& \leq \frac{C^{1}\left(g^{*}, h^{*}\right)-(1-\beta)(1-\alpha) \cdot C^{1}\left(g^{*}, h^{*}\right)}{C^{1}\left(g^{*}, h^{*}\right)} \\
& =\alpha+\beta-\alpha \beta .
\end{aligned}
$$

Here the inequality follows by Lemma 3.1. We now get:

$$
\begin{aligned}
C^{\beta}\left(g^{*}, h^{*}\right) & \leq(\alpha+\beta-\alpha \beta) \cdot C^{1}\left(g^{*}, h^{*}\right) \\
& \leq(\alpha+\beta-\alpha \beta) \cdot C^{1}\left(g^{\beta}, h^{\beta}\right) \\
& \leq \frac{\alpha+\beta-\alpha \beta}{\beta} \cdot C^{\beta}\left(g^{\beta}, h^{\beta}\right) \\
& =\left(1+\alpha \frac{1-\beta}{\beta}\right) \cdot C^{\beta}\left(g^{\beta}, h^{\beta}\right) .
\end{aligned}
$$

Here the first inequality follows by (2), the second by optimality of $\left(g^{*}, h^{*}\right)$ for $\beta=1$, and the third by Lemma 3.2

Both SLF and SCALE possess the property that the flow $g_{e}$ assigned on edge $e$ is bounded by $g_{e}^{*}+h_{e}^{*}$. We now prove that given this property, we can obtain constant upper bounds on the performance of the algorithm. We explicitly compute the bound as an expression for the case of $l_{e}(x)=a_{e} x+b_{e}$ and as a numerical value for specific values of parameters $\alpha, \beta$. 
We begin our analysis with the following technical lemma.

LEMMA 3.4. For any given initial solution $\left(g^{*}, h^{*}\right)$ and for a nonuser flow $h$ that is a Wardrop equilibrium (after fixing $g^{*}$ for the user flow), we get:

$$
\sum_{e \in E}\left(g_{e}^{*}+\beta h_{e}\right) \cdot l_{e}\left(g_{e}^{*}+h_{e}\right) \leq \sum_{e \in E}\left(g_{e}^{*}+\beta h_{e}^{*}\right) \cdot l_{e}\left(g_{e}^{*}+h_{e}\right) .
$$

Proof. Since $h$ is an equilibrium flow over $g^{*}$, we apply the variational inequality (1), to get:

$$
\sum_{e \in E} h_{e} \cdot l_{e}\left(g_{e}^{*}+h_{e}\right) \leq \sum_{e \in E} h_{e}^{*} \cdot l_{e}\left(g_{e}^{*}+h_{e}\right) .
$$

Multiplying both sides by $\beta$ and adding:

$$
\sum_{e \in E} g_{e}^{*} \cdot l_{e}\left(g_{e}^{*}+h_{e}\right)
$$

to both sides completes the proof.

We apply the $(\lambda, \mu)$-smoothness technique of [15] to get our upper bound on the performance of our algorithms.

Theorem 3.5. Suppose $\lambda \geq 1$ and $\mu \in(0,1)$ are numbers such that:

$$
(1+r)^{d} \leq \lambda \beta+\mu \beta r^{d+1}, \text { for every } r \geq 0
$$

Let $\left(g^{*}, h^{*}\right)$ be a given initial solution and $h$ an equilibrium flow for the non-users, given $g^{*}$. Then:

$$
\frac{C^{\beta}\left(g^{*}, h\right)}{C^{\beta}\left(g^{*}, h^{*}\right)} \leq \frac{\lambda}{1-\mu} .
$$

Proof. Let $f_{e}^{*}=g_{e}^{*}+h_{e}^{*}$. For every $e \in E$ with $f_{e}^{*}>0$, by setting $r=h_{e} / f_{e}^{*}$ in (3) and then multiplying both sides by $f_{e}^{* d+1}$, we get:

$$
f_{e}^{*}\left(f_{e}^{*}+h_{e}\right)^{d} \leq \lambda \beta f_{e}^{* d+1}+\mu \beta h_{e}^{d+1} .
$$

The inequality actually holds for every $e \in E$, since it trivially also holds for $f_{e}^{*}=0$. Note that:

$$
\left(g_{e}^{*}+\beta h_{e}^{*}\right)\left(g_{e}^{*}+h_{e}\right)^{d} \leq f_{e}^{*}\left(f_{e}^{*}+h_{e}^{d}\right) .
$$

Also note that

$$
\lambda \beta f_{e}^{* d+1}+\mu \beta h_{e}^{d+1} \leq \lambda\left(g_{e}^{*}+\beta h_{e}^{*}\right) f_{e}^{* d}+\mu\left(g_{e}^{*}+\beta h_{e}\right)\left(g_{e}^{*}+h_{e}\right)^{d} .
$$

Combining (4), (5), and (6), we get that for every $e \in E$ :

$$
\left(g_{e}^{*}+\beta h_{e}^{*}\right)\left(g_{e}^{*}+h_{e}\right)^{d} \leq \lambda\left(g_{e}^{*}+\beta h_{e}^{*}\right) f_{e}^{* d}+\mu\left(g_{e}^{*}+\beta h_{e}\right)\left(g_{e}^{*}+h_{e}\right)^{d} .
$$

Since $\lambda \geq 1$, we also get that for every $e \in E$

$$
\left(g_{e}^{*}+\beta h_{e}^{*}\right) b_{e} \leq \lambda\left(g_{e}^{*}+\beta h_{e}^{*}\right) b_{e}+\mu\left(g_{e}^{*}+\beta h_{e}\right) b_{e} .
$$

Adding (7) with (8) and then summing over all edges $e$ we get:

$$
\sum_{e \in E}\left(g_{e}^{*}+\beta h_{e}^{*}\right) \cdot l_{e}\left(g_{e}^{*}+h_{e}\right) \leq \lambda \cdot C^{\beta}\left(g^{*}, h^{*}\right)+\mu \cdot C^{\beta}\left(g^{*}, h\right) .
$$

Combining with Lemma 3.4, we get:

$$
C^{\beta}\left(g^{*}, h\right) \leq \lambda \cdot C^{\beta}\left(g^{*}, h^{*}\right)+\mu \cdot C^{\beta}\left(g^{*}, h\right) .
$$

Rearranging the terms completes the proof.

Theorem 3.5 suggests that finding the worst case $r$ in inequality (3), assuming $\lambda$ and $\mu$ are fixed and then optimizing $\lambda, \mu$ gives good bounds on the performance of the algorithms. As an example, we go through the process for the case of affine delay functions, i.e., $d=1$.
THEOREM 3.6. For affine delay functions, the following expression yields an upped bound on the worst case approximation ratios of $S_{L F}$ and SCALE.

$$
\left(1+\frac{\alpha(1-\beta)}{\beta}\right) \min _{\mu \in(0,1)}\left\{\frac{1}{4 \beta^{2} \mu(1-\mu)}+\frac{1}{\beta(1-\mu)}\right\}
$$

Proof. For $d=1$, inequality (3) becomes:

$$
1+r \leq \lambda \beta+\mu \beta r^{2} \Rightarrow \mu \beta r^{2}-r+\lambda \beta-1 \geq 0 .
$$

By the first order condition, the $r$ that minimizes the left hand side is $r=(2 \mu \beta)^{-1}$. Substituting for this value of $r$ in the inequality and rearranging, gives us that $\lambda$ and $\mu$ should satisfy:

$$
\lambda \geq \frac{1}{4 \mu \beta^{2}}+\frac{1}{\beta}
$$

We set $\lambda$ equal to the right hand side to ensure we satisfy the inequality and then optimize $\lambda /(1-\mu)$ which is now the following single variable expression:

$$
\frac{\lambda}{1-\mu}=\frac{1}{4 \beta^{2} \mu(1-\mu)}+\frac{1}{\beta(1-\mu)} .
$$

Finally, we multiply by $1+\alpha(1-\beta) / \beta$ as per Theorem 3.3 to get the result.

An example bound for $\alpha=1 / 4$ and $\beta=1 / 2$ is as follows.

COROLlARY 3.7. The worst case approximation ratios of $S_{L F}$ and SCALE for weighted Stackelberg routing with affine delays, $\alpha=1 / 4$, and $\beta=1 / 2$ are at most 9.33 .

\section{EXPERIMENTAL EVALUATION}

In this section we use real road networks to construct instances of the Stackelberg routing problem and evaluate the performance of the algorithms described in Section 3. We show that the algorithms that use the heuristic solution for the weighted objective function (i.e., $\beta$-SCALE, $\beta$-DiRECT, $\beta$-LLF, and $\beta$-SLF) yield solutions that are significantly better for the platform's users than the ones induced by the algorithms that use the optimal solution for the unweighted objective function (SCALE, LLF, and SLF). We also quantify the tradeoffs between the overall social cost and the quality of service to users of the platform. In the following subsections, we describe the data used to construct our experimental instances, the exact process followed to conduct our experiments, and the main conceptual conclusions we reach. Finally, we present our results in detail.

\subsection{Data}

Graphs. We extract graphs from Open Street Maps [11] for the cities of New York, Tokyo, Bangalore, and Delhi. We assign cost functions to the edges using the functional form of the Bureau of Public Roads [10], exactly as in [5, 8]. Specifically we set:

$$
l_{e}(x)=\frac{0.6 t_{e}^{f}}{c_{e}^{4}} x^{4}+t_{e}^{f}
$$

where $t_{e}^{f}$ is the time needed to cross the edge when the road is empty, i.e., the free-flow travel time, and $c_{e}$ is the capacity of the street, defined as the number of lanes multiplied by the free-flow speed. 


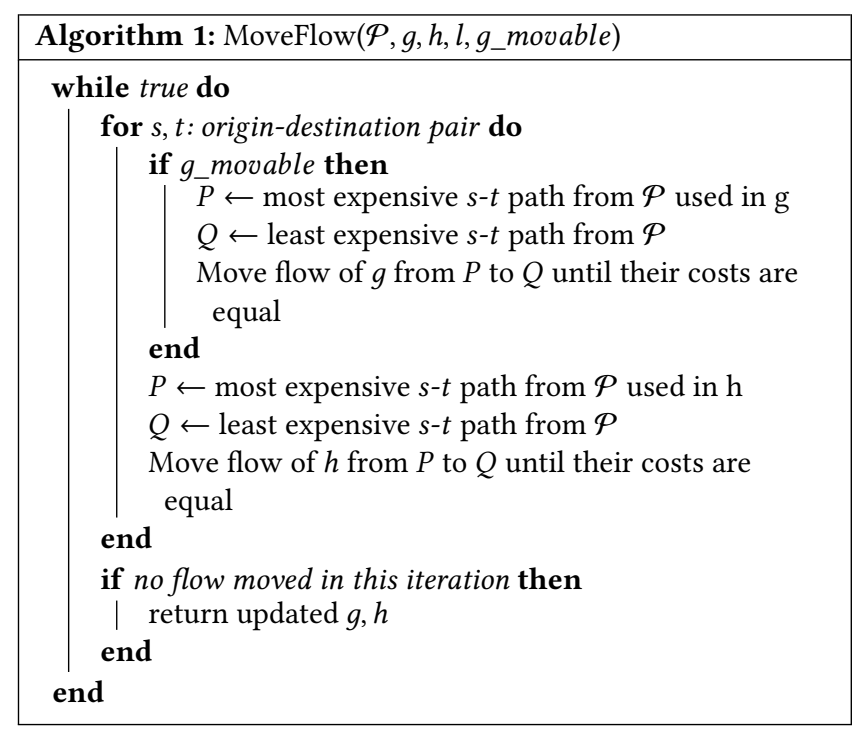

Traffic Flows. To generate traffic flows, we create uniformly random routing requests between points in the cities. We collect counts of navigation requests and aggregate them in clusters of size approximately 1 square $\mathrm{km}$. To tune our demands and make them realistic, we select characteristic routes in our cities and keep scaling the demands until the Wardrop equilibrium cost matches the empirically observed cost on the route at peak hour traffic. Such costs can be obtained through the standard interface of any navigation system, such as Google Maps.

Parameter Values. For each city and for each algorithm, we run the experiment for $\alpha$ and $\beta$ ranging from 0.2 to 0.8 in increments of 0.2 , taking all 16 such pairs of values for the parameters.

\subsection{Implementation Details}

Routing Strategies. For the purposes of efficiency and for the purposes of ensuring that drivers are routed on reasonable routes, we pre-construct a set of alternative routes between each pair of clusters. The number of such routes we produce is typically between 10 and 40 , depending on the distance between the origin and destination. These alternative routes are produced using the penalty method [2], which is the gold standard for producing alternative routes of high quality in road networks. The algorithm proceeds by iteratively producing a shortest path from origin to destination and applying a multiplicative penalty to each selected segment. The edge costs we use during alternative route computation are the free-flow costs, $l_{e}(0)$ for each $e$, and the multiplicative penalty we apply each time an edge is used is 1.2 .

Flow Computation Algorithm. Implementing our algorithms involves solving the optimization or Wardrop equilibrium computation version of flow assignment problems, as described in Sections 3.1 and 3.3. In those sections we gave theoretically robust algorithms to compute such solutions. In practice, however, we implement local search algorithms that quickly reach the corresponding solutions. These local search procedures rely on the MoveFlow algorithm described in Algorithm 1. The algorithm takes as input the set of

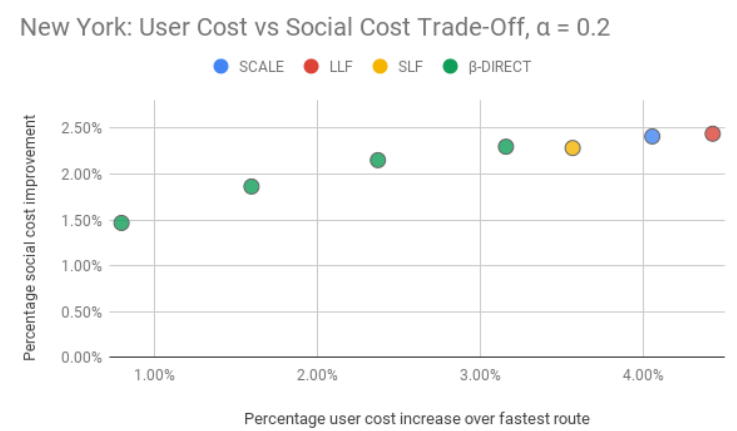

Figure 1: Trade-off between the user cost increase and the social cost improvement in New York City for standard Stackelberg algorithms and for our $\beta$-DIRECT. The four points for $\beta$-Direct correspond to $\beta$ ranging from 0.2 to 0.8 with 0.2 sized increments. $\beta$-Direct significantly decreases the excess user cost with a small loss in total cost gains.

paths $\mathcal{P}$, which gives all candidate paths between each $s$ - $t$ pair, the user flow $g$, the non-user flow $h$, the cost functions $l=\left\{l_{e}(), e \in E\right\}$, and a boolean variable $g \_$movable that declares whether flow $g$ is allowed to be moved. This last parameter is true when the algorithm computes optimal solutions and false when it computes Wardrop equilibria for $h$ over the Stackelberg flow $g$.

What we pass as latency functions to the algorithm determines what it finally outputs. If we input the exact delays $l_{e}(x)$, the algorithm will output a Wardrop equilibrium, since it will be moving flows to the fastest routes at each iteration. If we input the social $\operatorname{cost} x \cdot l_{e}(x)$, the algorithm will compute the optimal solution. If we input the weighted social cost:

$$
g_{e} \cdot l_{e}\left(g_{e}+h_{e}\right)+\beta h_{e} \cdot l_{e}\left(g_{e}+h_{e}\right),
$$

the algorithm will heuristically compute a near optimal solution with respect to the weighted objective function. These are all the variants we need to run our experiment.

\subsection{Summary of Findings}

Our experimental evaluation places the inefficiency of the Wardrop equilibrium solution when compared to the optimal traffic flow assignment at $7 \%$ for Tokyo, $10 \%$ for Bangalore, $13 \%$ for Delhi, and $14 \%$ for New York City. This is in effect the headroom that is available for Stackelberg algorithms. For any fixed value of $\alpha$, we observe that the traditional algorithms SCALE and LLF are the ones that offer the highest improvement to the social cost, however, at a significant price in terms of the cost of the platform's users. Specifically, for smaller values of $\alpha$ (which are also the most realistic ones), the travel times of the platform's users deteriorate (even if slightly) over the Wardrop equibrium, i.e., they get worse due to the platform's interventions. Moreover the users' costs are typically up to $8 \%$ larger than non-users' costs. These two facts raise the question whether these traditional algorithms are applicable in practice.

On the other hand, the algorithms that use the $\beta$-heuristic solutions, such as $\beta$-Direct, $\beta$-SCALE, $\beta$-LLF, and $\beta$-SLF, in almost all 


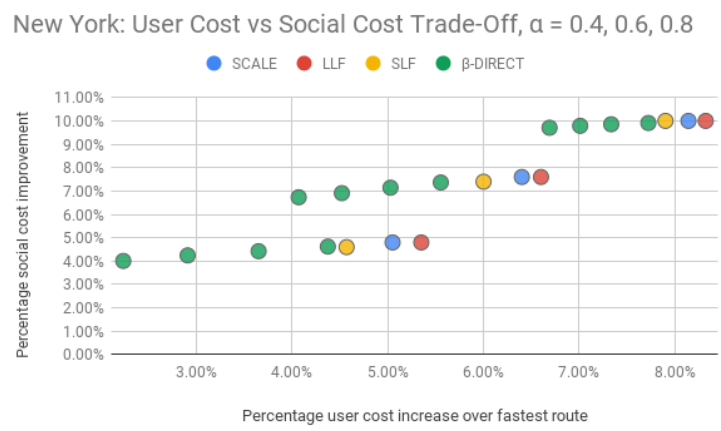

Figure 2: The same plot as Figure 1 for multiple other values of the penetration rate $\alpha$. The top cluster corresponds to $\alpha=0.8$, the middle to 0.6 , and the bottom to 0.4 . In all three clusters we observe $\beta$-DIRECT significantly decreasing the excess user cost with a small loss in total cost gains.

cases offer an improvement over the Wardrop equilibrium cost for the users, and, for small values of $\beta$ significantly limit the difference between user and non-user costs. (Note that non-user costs will always be at most the user costs for any algorithm since the non-user flow finds fastest paths and uses them.) Naturally, as $\beta$ increases the algorithms approach their unweighted counter-parts, since for $\beta=1$ the various versions collapse, e.g., 1-SCALE is the same as SCALE, 1-LLF is the same as LLF, etc.

In terms of comparing between our algorithms that use the $\beta$ weighted initial solution, we observe that they are typically close in terms of performance and that $\beta$-LLF appears to be the best one in almost all scenarios, with the exception of the low penetration regime in New York City, where $\beta$-DiRECT is slightly better.

Overall we observe that the findings and various plots are consistent across cities with slight perturbations. The main message coming out of our work is most cleanly presented in Figure 1 which showcases the example of $\alpha=0.2$ in New York City. The $x$-axis has the average percentage increase in the user cost over the non-user cost (which is the same as the average cost of the fastest available path over all drivers) and the $y$-axis has the percentage decrease in total travel time. We plot the three points that correspond to the standard algorithms SCALE, LLF, and SLF, and the four points that correspond to $\beta$-Direct for $\beta$ equal to $0.2,0.4,0.6$, and 0.8 . Note that for $\beta=1$ it will coincide with Scale. We see that $\beta$-Direct dominates SLF offering a higher improvement in the social cost with a lower cost overhead for users. We also see that with a small sacrifice in overall cost we can get a significant improvement in terms of user overhead. Figure 2 presents the same plots for $\alpha$ equal to $0.4,0.6$, and 0.8 in a single image. Finally, Figure 3 presents how varying $\alpha$ impacts the algorithms. We observe that all algorithms tend to improve in a near linear fashion as the penetration rate $\alpha$ increases.

\subsection{Cities}

Figure 4 presents the results of our experimental evaluation with a low penetrations rate, $\alpha=0.2$, in New York City. The top image shows the percentage improvement achieved for the social cost for
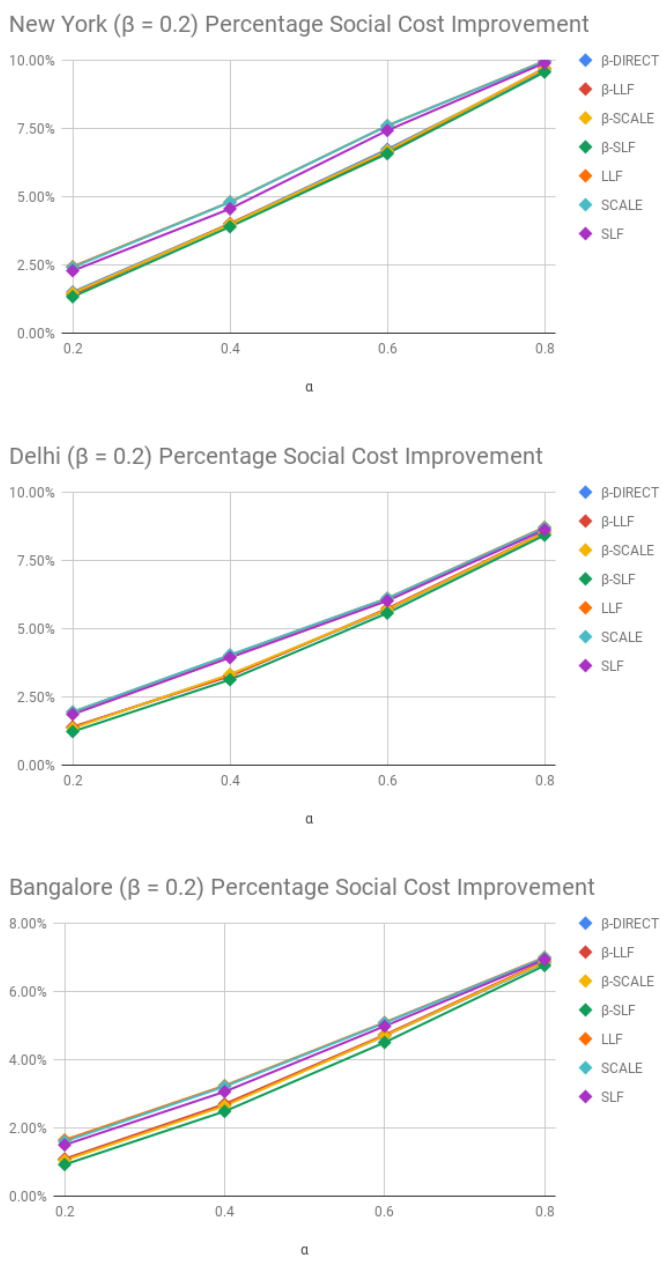

Figure 3: The impact of $\alpha$ on the performance of the algorithms. All algorithms tend to recover the available headroom in a near linear fashion.

each algorithm as $\beta$ varies. Note that ScAle, LlF, and SlF do not depend on $\beta$ and are, hence, constant. The middle image shows the percentage user excess cost, meaning the average percentage increase for the user average cost over the average non-user score, which coincides with the average fastest path cost. The third image combines the previous two in a bang-per-buck plot, which shows the ratio of the previous two point-wise, i.e., the percentage decrease in the social cost over the percentage user excess score. Figure 5 presents the same information at a high penetration rate regime in which $\alpha=0.8$

We observe that ScAlE is the best one in terms of traditional algorithms and the best one overall in terms of the social cost. However, it is the one that suffers most in terms of the excess cost for the platform's users. The $\beta$ dependent algorithms improve their cost nearly linearly with increases in $\beta$ and similarly degrade the user experience linearly as $\beta$ increases. The bang-per-buck plot show that increases in user cost are less valuable as $\beta$ increases. 

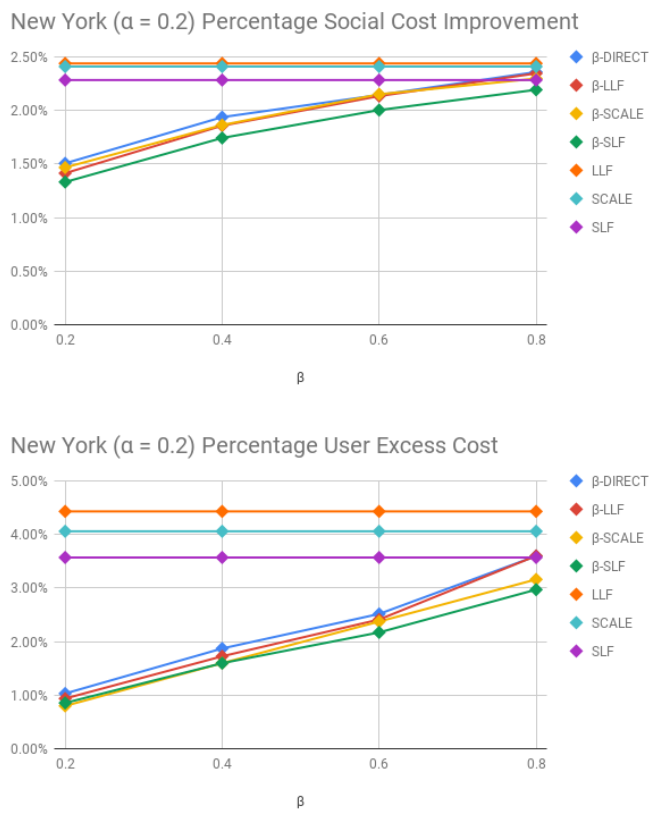

New York $(a=0.2)$ Social Cost Improvement Per User Excess

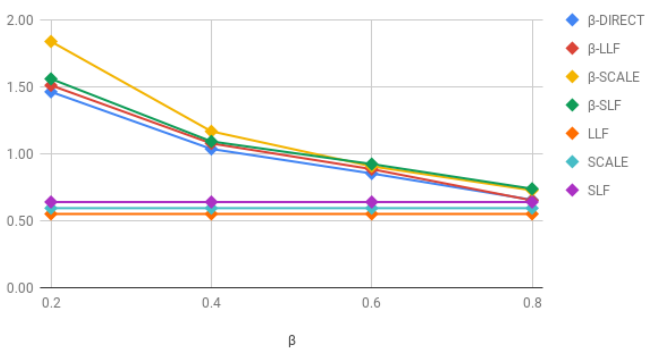

Figure 4: Experimental evaluation in New York with $\alpha=0.2$.

The same conclusion can be reached from Figures 1 and 2 as well. In terms of comparing between the $\beta$ dependent algorithms, we observe $\beta$-DIRECT and $\beta$-LLF performing slightly better than the others.

Figure 6 presents the results of our experimental evaluation with a low penetrations rate, $\alpha=0.2$, in Tokyo, whereas Figure 7 showcases $\alpha=0.8$. We observe similar conclusions as in New York City, with the only difference being that $\beta$-LLF is better for both the low and high penetration regime than all other $\beta$ dependent algorithms. Similarly, in the Appendix, Figures 8 and 9 present the evaluation for Bangalore and Figures 10 and 11 present the evaluation for Delhi.

\section{DISCUSSION}

In this work we presented the first data-driven evaluation for Stackelberg algorithms in road networks. We identified an important shortcoming of standard literature algorithms and addressed it through formulating the weighted Stackelberg problem. We proved that this problem is approximable within constant factors and used heuristics of the problem in real city evaluations.
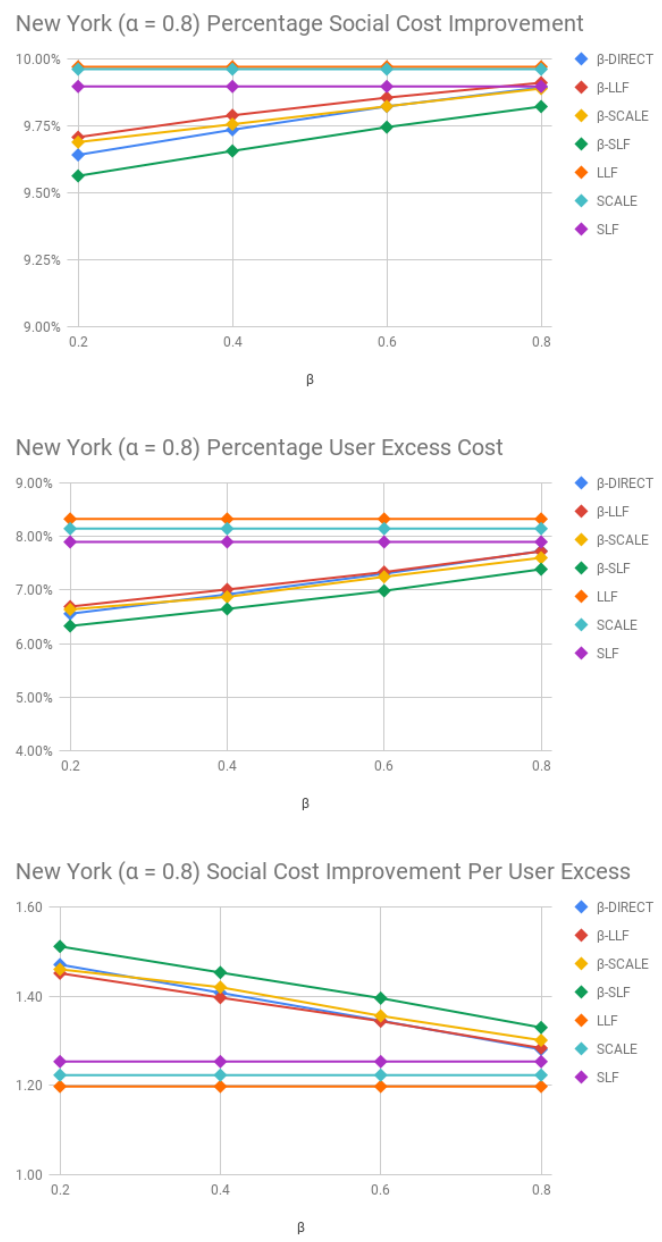

Figure 5: Experimental evaluation in New York with $\alpha=0.8$.

Our approach uses the well established Wardrop model of traffic in road networks. This model focuses on routing traffic as a flow. An important consideration is that in actual navigation systems requests arrive one by one and need to be routed in this fashion. Moreover, these requests have specific origins and destinations that are not simply centers of larger regions as in the Wardrop model. However, these differences between theory and practice are not hard to address. Once the platform has an understanding of current demand volumes, it can solve for the Stackelberg flow and then iteratively assign cars to the paths of the flow proportionally to the volume they carry. The exact location of the origin and destination can be addressed with local corrections of the path near its start and end.

There are various interesting problems that can follow up on our work. Here we seek to minimize the suboptimality of user paths through formulating the weighted Stackelberg problem. An interesting future direction is to route in a fashion that ensures the users are an $\epsilon$ factor away from their fastest path (i.e., form an $\epsilon$ Wardrop equilibrium) and seek to optimize for the social cost. 

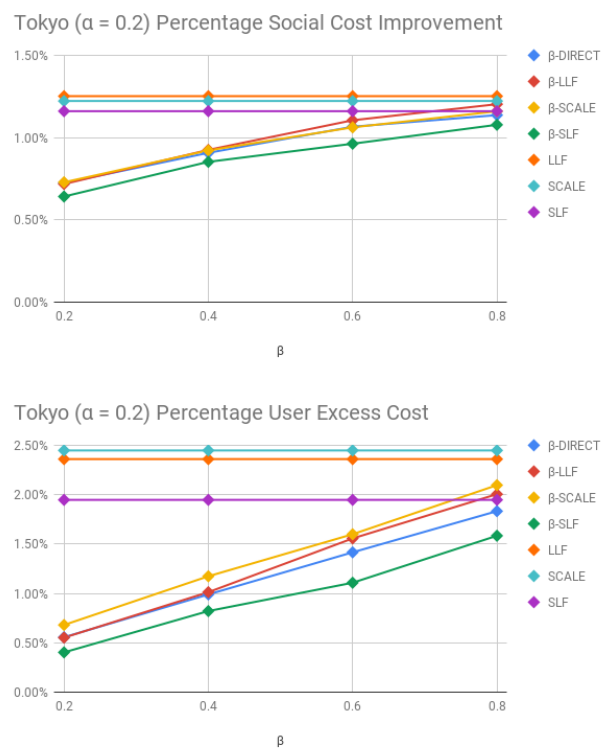

Tokyo $(a=0.2)$ Social Cost Improvement Per User Excess

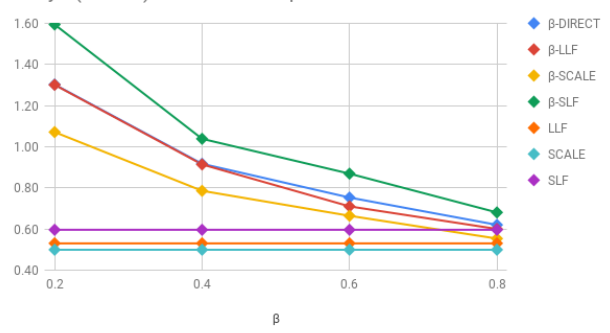

Figure 6: Experimental evaluation in Tokyo with $\alpha=0.2$.

\section{REFERENCES}

[1] Daniel Ayala, Ouri Wolfson, Bo Xu, Bhaskar DasGupta, and Jie Lin. 2011. Parking slot assignment games. In 19th ACM SIGSPATIAL International Symposium on Advances in Geographic Information Systems, ACM-GIS 2011, November 1-4, 2011 Chicago, IL, USA, Proceedings. 299-308.

[2] Roland Bader, Jonathan Dees, Robert Geisberger, and Peter Sanders. 2011. Alternative Route Graphs in Road Networks. In Theory and Practice of Algorithms in (Computer) Systems - First International ICST Conference, TAPAS 2011, Rome, Italy, April 18-20, 2011. Proceedings. 21-32.

[3] Francisco Benita, Vittorio Bilò, Barnabé Monnot, Georgios Piliouras, and Cosimo Vinci. 2020. Data-Driven Models of Selfish Routing: Why Price of Anarchy Does Depend on Network Topology. In Web and Internet Economics - 16th International Conference, WINE 2020, Beijing, China, December 7-11, 2020, Proceedings. 252-265.

[4] Vincenzo Bonifaci, Tobias Harks, and Guido Schäfer. 2010. Stackelberg Routing in Arbitrary Networks. Math. Oper. Res. 35, 2 (2010), 330-346.

[5] Serdar Colak, Antonio Lima, and Marta Gonzalez. 2016. Understanding congested travel in urban areas. Nature Communications 7, 10793 (2016).

[6] Richard Cole, Yevgeniy Dodis, and Tim Roughgarden. 2006. How much can taxes help selfish routing? 7. Comput. Syst. Sci. 72, 3 (2006), 444-467.

[7] Luca Foti, Jane Lin, Ouri Wolfson, and Naphtali David Rishe. 2017. The Nash Equilibrium Among Taxi Ridesharing Partners. In Proceedings of the 25th ACM SIGSPATIAL International Conference on Advances in Geographic Information Systems, GIS 2017, Redondo Beach, CA, USA, November 7-10, 2017, Erik G. Hoel, Shawn D. Newsam, Siva Ravada, Roberto Tamassia, and Goce Trajcevski (Eds.) ACM, 72:1-72:4.

[8] Barnabé Monnot, Francisco Benita, and Georgios Piliouras. 2017. Routing Games in the Wild: Efficiency, Equilibration and Regret - Large-Scale Field Experiments in Singapore. In Web and Internet Economics - 13th International Conference, WINE 2017, Bangalore, India, December 17-20, 2017, Proceedings. 340-353.
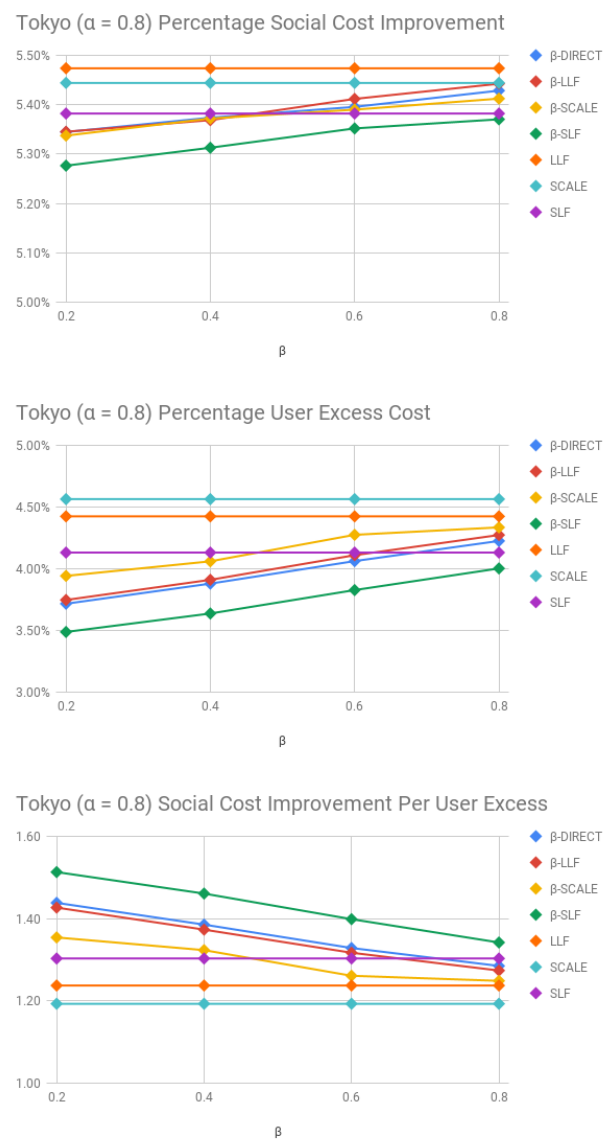

Figure 7: Experimental evaluation in Tokyo with $\alpha=0.8$.

[9] Lefteris Ntaflos, George Trimponias, and Dimitris Papadias. 2017. Game-Theoretic Solutions for Constrained Geo-Social Event Organization. In Proceedings of the 25th ACM SIGSPATIAL International Conference on Advances in Geographic Information Systems, GIS 2017, Redondo Beach, CA, USA, November 7-10, 2017. 69:169:4.

[10] Bureau of Public Roads. 1964. Traffic assignment manual. US Department of Commerce.

[11] OpenStreetMap contributors. 2017. Planet dump retrieved from https://planet.osm.org . https://www.openstreetmap.org.

[12] Arthur Cecil Pigou. 2013. The Economics of Welfare. Palgrave Macmillan.

[13] Tim Roughgarden. 2003. The price of anarchy is independent of the network topology. F. Comput. Syst. Sci. 67, 2 (2003), 341-364.

[14] Tim Roughgarden. 2004. Stackelberg Scheduling Strategies. SIAM f. Comput. 33, 2 (2004), 332-350.

[15] Tim Roughgarden. 2015. Intrinsic Robustness of the Price of Anarchy. F. ACM 62, 5 (2015), 32:1-32:42.

[16] Tim Roughgarden and Éva Tardos. 2002. How bad is selfish routing? f. ACM 49, 2 (2002), 236-259.

[17] Yosef Sheffi. 1985. Urban Transportation Networks: Equilibrium Analysis with Mathematical Programming Methods. Prentice-Hall.

[18] M. J. Smith. 1979. The marginal cost taxation of a transportation network. Transportation Research B: Methodological 13 (1979), 237-242. Issue 3.

[19] Chaitanya Swamy. 2012. The effectiveness of stackelberg strategies and tolls for network congestion games. ACM Trans. Algorithms 8, 4 (2012), 36:1-36:19.

[20] John Glen Wardrop. 1952. Some theoretical aspects of road traffic research. Proceedings of the Institute of Civil Engineers II, 1 (1952), 325-378.

[21] Hyejin Youn, Michael T. Gastner, and Hawoong Jeong. 2008. Price of Anarchy in Transportation Networks: Efficiency and Optimality Control. Phys. Rev. Lett. 101 (Sep 2008), 128-701. Issue 12. 


\section{A APPENDIX}

In this Appendix we include the results for the experimental evaluation in two additional cities: Bangalore and Delhi. Similarly to New York City and Tokyo, we include 3 plots for each city and value of $\alpha$ (either low-penetration $\alpha=0.2$, or high penetration $\alpha=0.8$ ). The first in each such collection of plots shows the percentage cost improvement for SCALE, LLF, and SLF, and, for $\beta=0.2,0.4,0.6,0.8$, the same value for $\beta$-SCALE, $\beta$-LLF, $\beta$-SLF, and $\beta$-Direct. Similarly, the second plot in each collection plots the percentage excess user cost over the fastest path (i.e., the non-user cost), and the third plot the percentage cost improvement per percentage user excess. We observe that the results reproduce the same conclusions as in New York City and Tokyo.
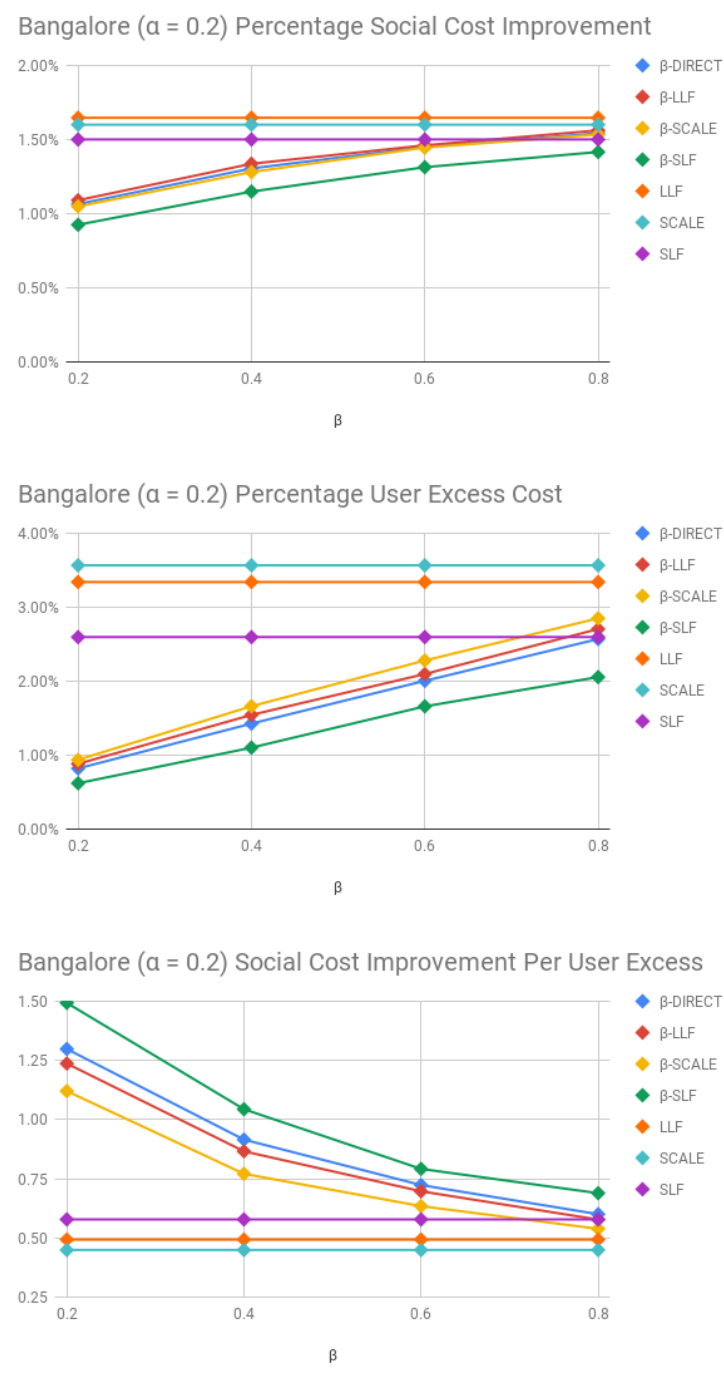

Figure 8: Experimental evaluation in Bangalore with $\alpha=0.2$.
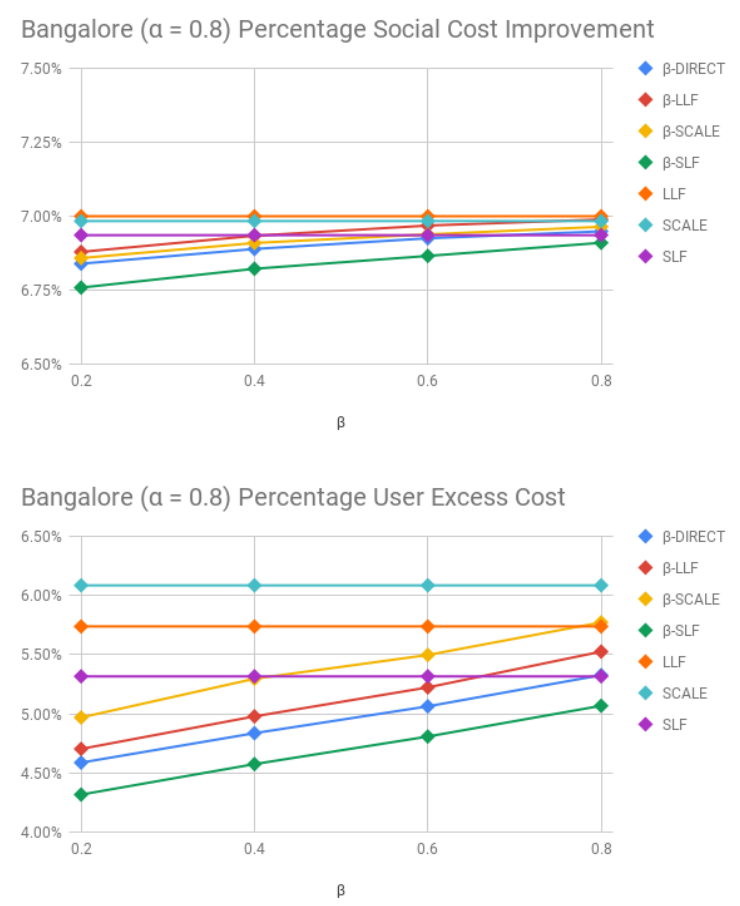

Bangalore $(a=0.8)$ Social Cost Improvement Per User Excess

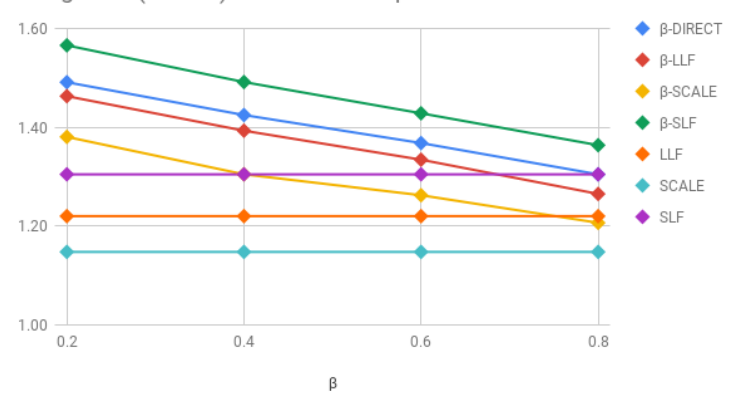

Figure 9: Experimental evaluation in Bangalore with $\alpha=0.8$. 


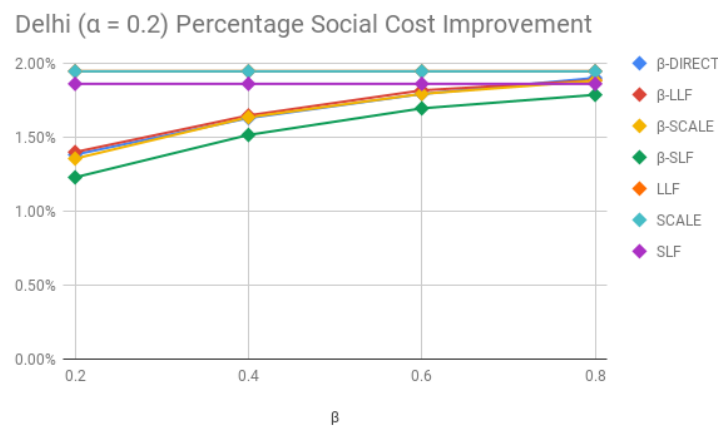

Delhi $(a=0.2)$ Percentage User Excess Cost

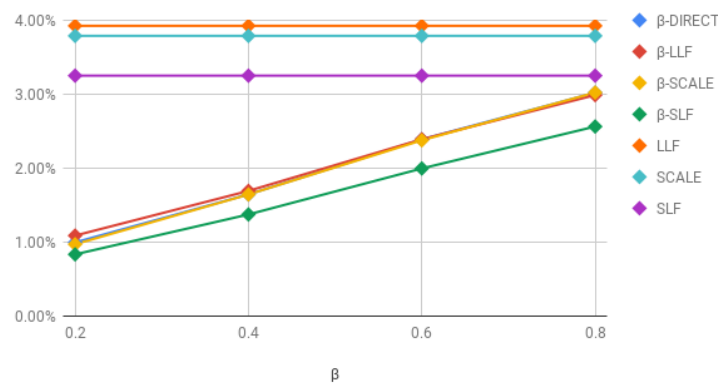

Delhi $(a=0.2)$ Social Cost Improvement Per User Excess

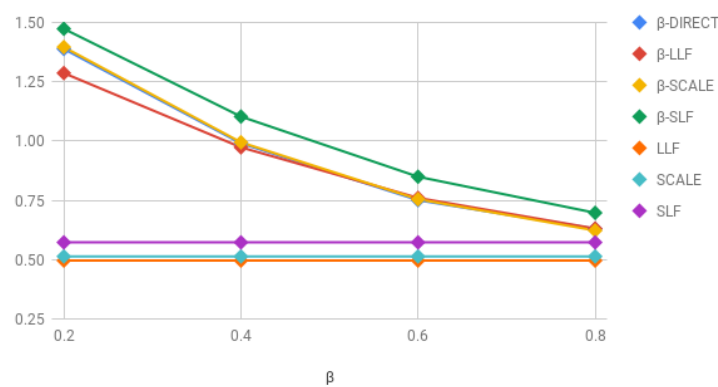

Figure 10: Experimental evaluation in Delhi with $\alpha=0.2$.

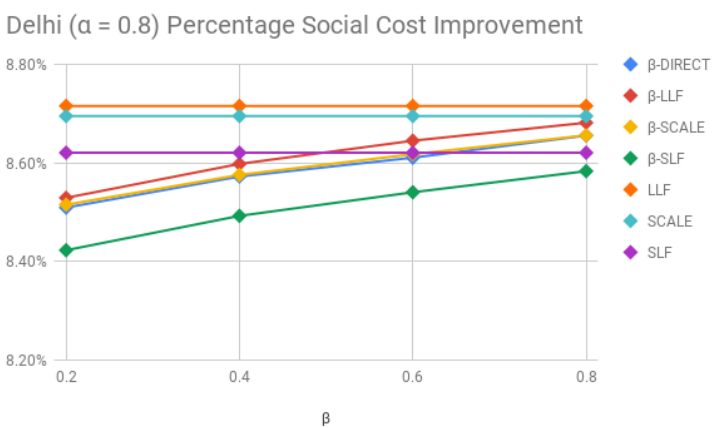

Delhi $(a=0.8)$ Percentage User Excess Cost

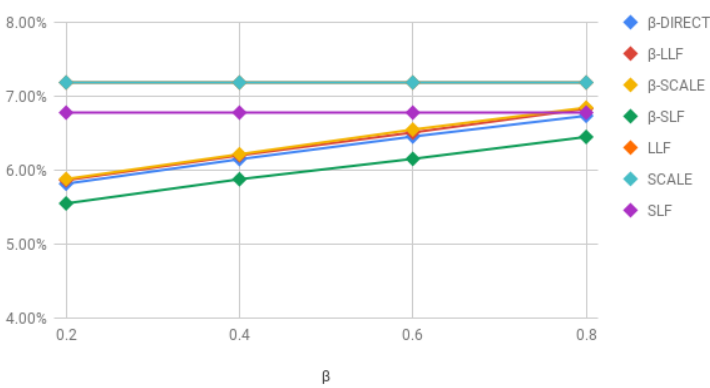

Delhi $(\alpha=0.8)$ Social Cost Improvement Per User Excess

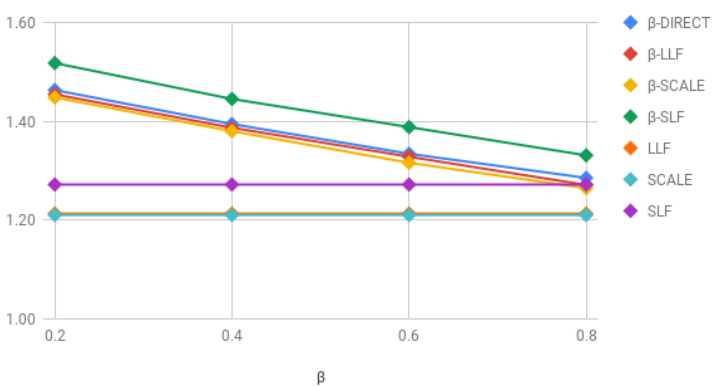

Figure 11: Experimental evaluation in Delhi with $\alpha=0.8$. 